Copyright (C) 2013 IEEE. Personal use of this material is permitted. Permission from IEEE must be obtained for all other uses, in any current or future media, including reprinting/republishing this material for advertising or promotional purposes, creating new collective works, for resale or redistribution to servers or lists, or reuse of any copyrighted component of this work in other works. 


\title{
Instrumentation for safe vehicular flow in intelligent traffic control systems
}

\author{
using wireless networks
}

\author{
Halit Eren, Hariani Ma’tang Pakka, Ahmed S. AlGhamdi, and Yizuo Yue \\ Department of Electrical and Computer Engineering \\ Curtin University, Perth, WA, Australia \\ h.eren@,curtin.edu.au
}

\begin{abstract}
This paper describes a ZigBee based wireless system to assists traffic flow on arterial urban roads. Real-time simulation in laboratory environment is conducted to determine the traffic throughput to avoid possible congestions or ease existing congestions. Random numbers are generated to mimic approaching traffic, and this information is shared by a ZigBeebased real-time wirelessly network. Wireless nodes are connected to different PLCs representing different traffic lights in a cluster. Once the information is shared the timing and sequencing decisions are taken collectively in a synchronized manner. In this paper, the information is displayed on SCADA connected to each PLC for viewing the characteristics of continuous vehicular flow. It is found that the topology of the network can play an important role in the throughput of data, which may be critical in safety critical operations such as the control of traffic lights. This paper aims to highlight some of the possible effects of dataflow flow and time-delays faced by modern intelligent control of traffic lights.
\end{abstract}

Keywords-wireless; sensor networks; ZigBee; vehicular; traffic flow; SCADA, sensors, simulation

\section{INTRODUCTION}

As the urbanization continues to grow all around the world, the number of vehicles in cities and associated traffic problems increase, often leading to unacceptable levels of traffic congestions. These traffic congestions are a major concern for the commuters, the public, as well as traffic authorities, and the governments. In many urban areas, existing methods for traffic management, and surveillance and control are not sufficient in terms of performance, cost, maintenance, and support. Among many other reasons, one important factor causing the congestion is the nonsynchronous operations of traffic lights. Hence synchronous operation concept is worth studying, since it can offer at least a partial solution to ease traffic problems. It also can lead to an efficient management of traffic lights to assist continuous flow of vehicles with minimum time delays.

According to National Centre for Policy Analysis, USA, (http://www.ncpa.org/sub/dpd/index.php?Article ID=1119 ) [1] "The U.S. motoring public pays an estimated $\$ 123$ billion annually in lost time, wasted fuel, and other vehicle operation costs, plus billions more for high-tech electronics to moderate America's intersections" and "In addition, traffic signals cause congestion, because they don't moderate the use of the intersection efficiently: often cars sit idly at a red light only to have no traffic using the green light. Overall, about 40 percent of vehicle delays in urban areas are due to traffic signal inefficiencies."
Congestion concerns coupled with the accidents, caused by traffic lights, have accelerated intelligent traffic management systems in many urban areas as exemplified in reference [2].

In modern applications, the wireless sensor network (WSN) is finding extensive use in traffic monitoring, control and management. Nevertheless it is worth mentioning that wireless methods have been applied in traffic flow and control for decades. One of the first applications was reported in 1956 by Sheffield [3]. In his paper, a VHF radio system was used to provide communications for both control and voice transmission purposes in a railway operation with a distance of up to $120 \mathrm{~km}$. In $70 \mathrm{~s}, 80 \mathrm{~s}$ and $90 \mathrm{~s}$ radiolocation became a popular practice to monitor and control ships, aircrafts, and land vehicles [4]. Nowadays, the use of wireless networks in traffic monitoring and control is very common implemented in many countries $[5,6,7,8]$.

However, wireless networks come with their own challenges, such as energy consumption, synchronisation of the network nodes, timing, data flow rate, errors, throughput, and range $[9,10]$. In many applications, special methods must be designed to overcome some of these problems.

In this paper, we aim for a solution to traffic congestions on the main roads by synchronizing traffic lights in a safe manner; thus allowing flow of vehicles in a steady elastic mode. It is aimed that each traffic light is aware of the traffic load in other intersection and adapt to new situation by changing its own sequencing. The decisions are taken collectively with the other lights to ensure a continuous flow.

In this study, the following steps have been implemented: Firstly, the traffic flow has been modelled mathematically. Secondly, the cycle length of each traffic light has been determined, which consists of the average distance between intersections and the vehicular flow on the road. Thirdly, suitable programmable logic controllers (PLCs) programs have been developed in conjunction with the theoretical requirements. A supervisor control and data acquisition (SCADA) programs have been designed and implemented to monitor and control of the PLCs. And finally, the communication between the SCADA systems and PLCs has been realized by wireless network using ZigBee technology. It has been shown that PLC/SCADA combination is flexible, convenient to use, and has a potential to be used in intelligent traffic control systems. This work is continuing by adding more intersections with larger synchronization needs leading to improved modelling of the system. 


\section{TRAFFIC FLOW CONSIDERATIONS}

Traffic congestion is said to appear when many vehicles attempt to use a common transportation infrastructure with a limited capacity $[11,12,13]$. As a result, traffic congestion takes place creating problems, as

- Vehicle queuing causing delays in the movement of people and goods.

- Idling of engines that wastes scarce resources and increases environmental pollution.

- Reduction in safety due to various human errors.

- Decreases satisfaction in residential areas due to noise and pollution.

In order to address some of these problems, the traffic flow has been studied by many researchers using mathematical models expressed in terms of the relationships between three main components. These being

1. Flow rate - determined by the number of vehicles passing through a given section or a point of the road in a fixed duration.

2. Density - found by the total number of vehicles occupying a particular length of road.

3. Velocity - determined by the rate of motion of a vehicle or aggregate of vehicles as distance travelled per unit time, usually in kilometres per hour.

Taking these three factors, the flow of vehicles can be investigated by using many different models, such as elastic band strings theory that contracts and expands continuously, and dynamic traffic cellular automata (DTCA) model [14, 15]. For example, between the consecutive traffic lights, vehicles will follow each other depending on factors such as driver behaviour, vehicle dynamics, road conditions, and the number of vehicles in the string. In this paper, the traffic flow is investigated using DTCA model in which vehicles travel as a string in stretched mode with certain speeds. The traffic lights should be synchronised in such a way that the string of vehicles continues their journey without having to stop at the consecutive lights. To achieve this objective, there must be continuous information flow between the traffic lights, which are simulated at a set of PLC stations, and the communication takes place between the PLCs through a ZigBee wireless network.

Programmable logic controllers are used to control industrial processes and machines. They can be programmed to execute instructions for communications, data manipulations, arithmetic operations, counting, timing, sequencing, and so on. They are robust, cost effective devices, and can be programmed and reprogrammed easily by using various programming languages; either in text or graphical formats. The use of PLCs in traffic light control applications have been reported in various studies [16] The SCADA system, on the other hand, can be defined as the combination of telemetry and data acquisition systems primarily aiming human-machine interaction (HMI). Remote terminal units (RTUs) are used to gather the information from the processes. The collected information is generally transferred to a master terminal unit (MTU) for further processing, decision making, and control of processes. SCADA is capable of carrying out significant data analysis, implement control commands, and display the information in numerous forms for operators to observe and control the system from a central control room.

Traffic flow" can be defined as the study of the movement of individual drivers and vehicles between two points [17, 18]. Vehicular flow on the other hand is the measure of vehicles per time period from a point. It is difficult to conduct an accurate study of traffic flow because the behaviour of individual driver cannot perfectly be predicted. However, it is fair to say that most drivers are likely to behave in a similar manner consistently under normal conditions. After some assumptions their behaviour can be expressed in various mathematical forms [19].

Transportation systems can be made efficient and safe by improving the control of the signal durations at the traffic lights $[20,21]$. The procedures include: schedule and control to achieve satisfactory performance, synchronisation, adaptable cycles at successive intersections, intersection's cycle time, and colour sequence of lights at each cycle.

Modelling of traffic flow is greatly affected by the synchronisation issues associated with the conflict resolution and resource sharing. In order to control these processes, various artificial intelligence techniques and mathematical methods have been suggested [22, 23].

Once we consider all these factors the traffic flow becomes a very complex multidimensional problem, some components can easily be described deterministically and whereas other affecting components will have rare occurrences, probabilities, and uncertainties.

\section{LABORATORY SETUP OF TRAFFIC FLOW AND WIRELESS SYSTEM}

Using PLCs for traffic lights control has been investigated in various other studies [24, 25]. Wireless networks have been used in urban traffic control extensively and many methods have been reported. The cellular digital packet data (CDPD), and the IEEE 802.11-based wireless systems are some examples $[5,6,7,8,26,27]$ on the applications of wireless techniques. In this paper, the ZigBee wireless communication technology has been selected, since it allows the communications of PLCs and SCADA system effectively and efficiently. In addition, ZigBee has unique characteristics such as flexibility is setting up and usage, fault tolerance, low cost, and rapid deployment, which make it feasible in traffic flow applications [28].

In this study, a system was setup as shown in Figure 1.

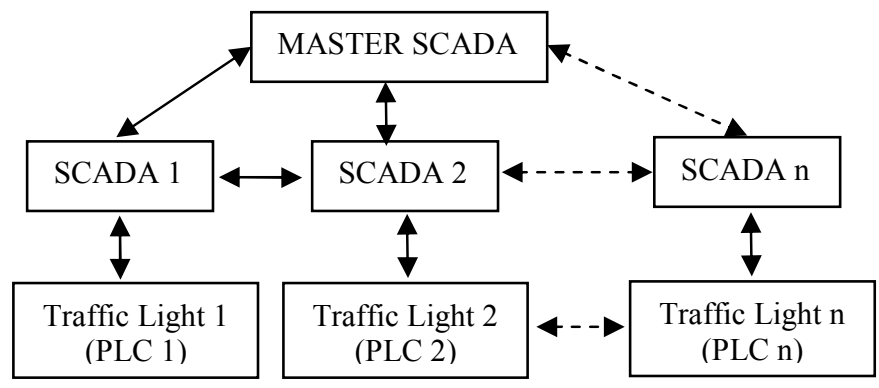

Fig. 1. Wireless system set up 
Each PLC station is programmed to mimic the control the traffic light sequences at a junction. For wireless communications, the ZigBee hardware and software has been used for transferring information between PLC-to-SCADA and SCADA-to-SCADA stations. The wireless system hardware selected is XBee modules from Digi International. The system has been setup in various different network configurations to investigate and select the best topology for information flow with minimum time delays and maximum throughputs.

\section{SYSTEM IMPLEMENTATION AND RESULTS}

A typical arterial control system, considered in this study, is comprised of a connected stretch of road and adjacent signalised intersections. According to Zhang [23], arterial street control revolves around establishing an efficient relationship between the beginning of the green light at one intersection and the beginning of green light at the next intersection. These types of considerations are essential such that each wave of traffic can aim to catch a green signal as soon as vehicles arrive to the next signalised intersection. Ultimately, the delays can be reduced due to the nonstop flow of traffic for that particular cluster of vehicles.

Orderly self-organising traffic flow is only possible if there is adequate information on the arrival and departure of vehicles, vehicle detection technology, and counting of arrivals at the intersections. Several methods are available for this purpose, including sensors based on light detection, cameras, areal sensing, or conventional ground detectors using magnetic sensors [26, 27, 28, 29, 30]. Once an adequate operational sensing method is developed, various artificial intelligence and adaptive techniques can be employed [31,32].

Three sets of traffic lights on a stretch of road representing an arterial highway have been shown in Figure 2.

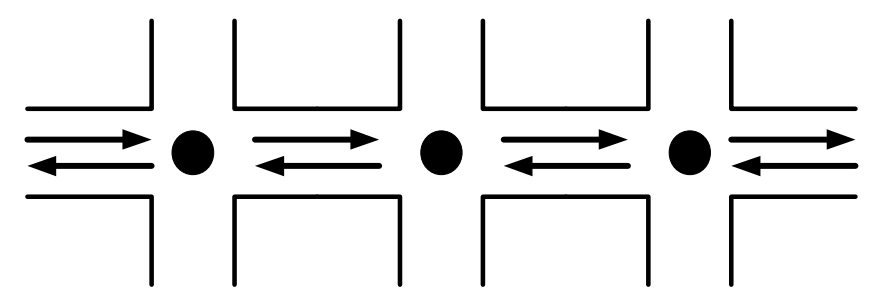

Fig. 2. Representation of a typical arterial road.

To determine requirements for a 3-traffic lights plan, the following calculations are carried out

1. Cycle lengths of lights.

2. Starting time of the cycles.

3. Proportional durations of red, amber, and green cycle.

The time that it takes to travel a cluster of vehicles from one intersection to the next can be calculated. This determines the beginning and durations of cycle at the next set of traffic lights. This can be determined by a simple formula, as

$$
T=\frac{d}{v}
$$

where $d$ is average distance between two intersection pairs, $v$ is average velocity vehicles.
Keeping in mind the flow in each direction, should adapt the proportion of red and green length at every junction. If $\varphi$ and $\psi$ denote the two average flows arriving at the junction and $\tau$ and $T-\tau$ the length of the corresponding green phase we must have

$$
\varphi /(\varphi+\psi)=\tau / T
$$

With similar assumptions and theoretical approaches, the operational principles must suitably be converted to appropriate PLC programs and sequential timing parameters must be determined. A typical sequence is

- Cycle length for all three intersections is equal to $120 \mathrm{~s}$.

- Phase difference between each of the two intersection cycle is $60 \mathrm{~s}$.

The length of the green phases in each intersection typically is set as follows:

- The straight direction in the main road is equal to $60 \mathrm{~s}$ at a specified vehicle speed.

- The straight direction in the other road is equal to $32 \mathrm{~s}$.

- The left directions in all roads are equal to $12 \mathrm{~s}$.

Based on these, and similar timing sequences, the PLC programs at each traffic light is developed, and linked to a SCADA system via the ZigBee wireless network. Also, each PLC is able to communicate with the others using various network topologies, namely mesh, star, and tree topologies.

Random numbers were generated at each station to represent the number of arriving vehicles at that intersection at a particular time. A Citect/SCADA operating interface system (OIS) is developed and implemented to monitor and control each traffic light. A typical SCADA display is illustrated in Figure 3. SCADA is aware of vehicles at the local traffic light as well as the vehicles at the nearby lights. It modifies the traffic light sequences for optimum flow.

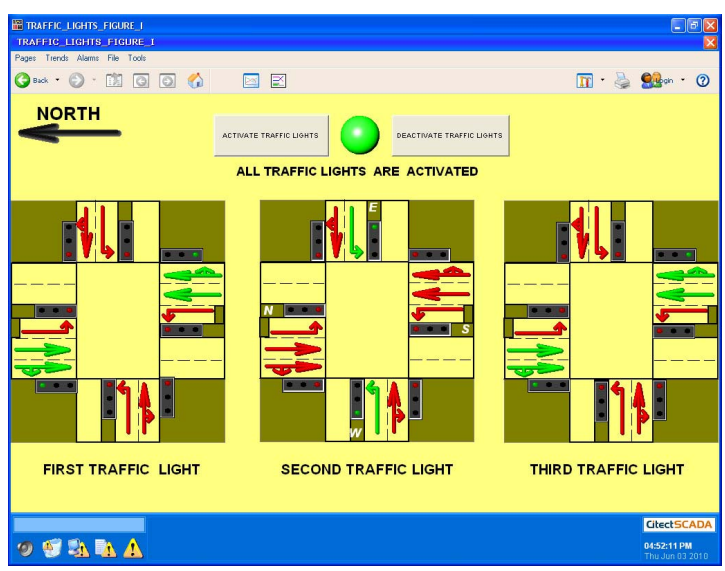

Fig. 3. A typical SCADA display.

\section{WIRELESS SYSTEM SIMULATION}

The IEEE 802.15.4 standard for ZigBee specifies the physical layer and the MAC sub-layer for low-rate wireless personal area networks (LR-WPANs). Here, the physical layer operating in the $2.4 \mathrm{GHz}$ frequency band with $250 \mathrm{kbps}$ of bit rate. The MAC sublayer supports the beacon-enabled or 
nonbeacon-enabled operational modes that may be selected by a central controller of the personal area network (PAN), called PAN coordinator (PANC). Here, the media access is a contention based system using beacon-enabled mode.

The operation of the ZigBee networks as applied to the traffic light operation has been simulated on OPNET. The OPNET is a commercial network simulator for simulation of a wide range of wireless technologies as well as wired systems. It has graphical packages and libraries for presenting simulation scenarios and results. In thus study, the basic three phases of OPNET is deployed. That is: node models are selected and configured. Network set up connections are organised for different entities. And, the desired statistics (e.g., local or global) are selected [33]. Figure 4 shows a typical system set up, each end-device representing a traffic light.

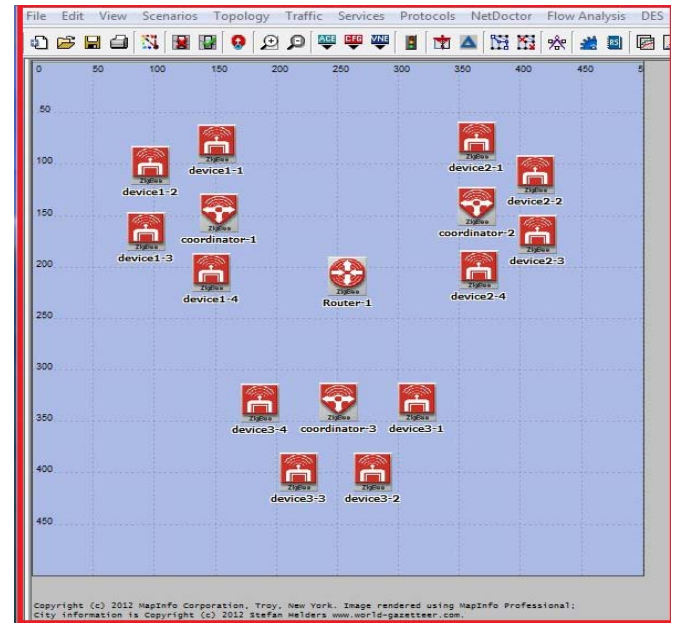

Fig. 4. Wireless network setup - each end device represents a set of traffic lights.

Simulations indicate that flow of critical information depends on type network topology and power level at the transmitter. Figure 5 shows throughput for different topologies.

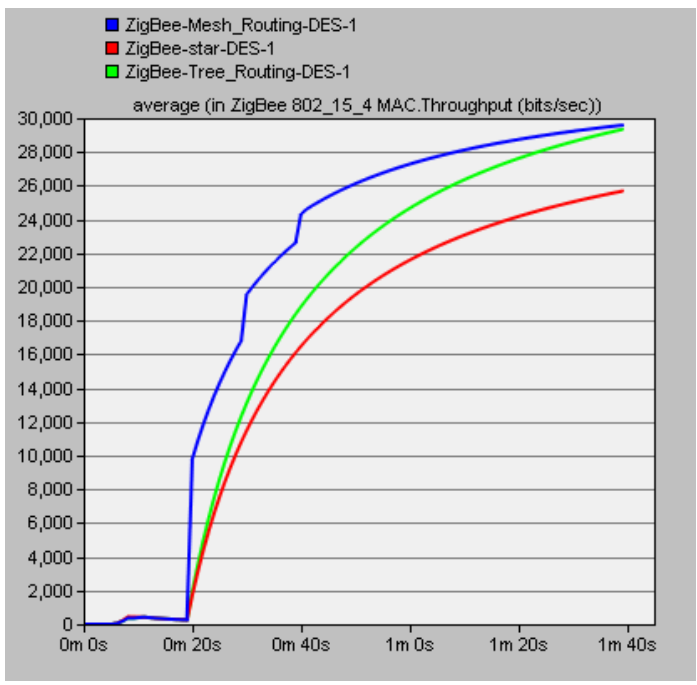

Fig. 5. ZigBee MAC layer throughputs (bits/sec)
There are also time delays present particularly in the initial stages of the network configuration, which is dependent on the network topology, as illustrated in Figure 6. These delays are very important, particularly in heavy traffic conditions, where precise timing of individual lights and timely information flow between sets of traffic lights can be critical.

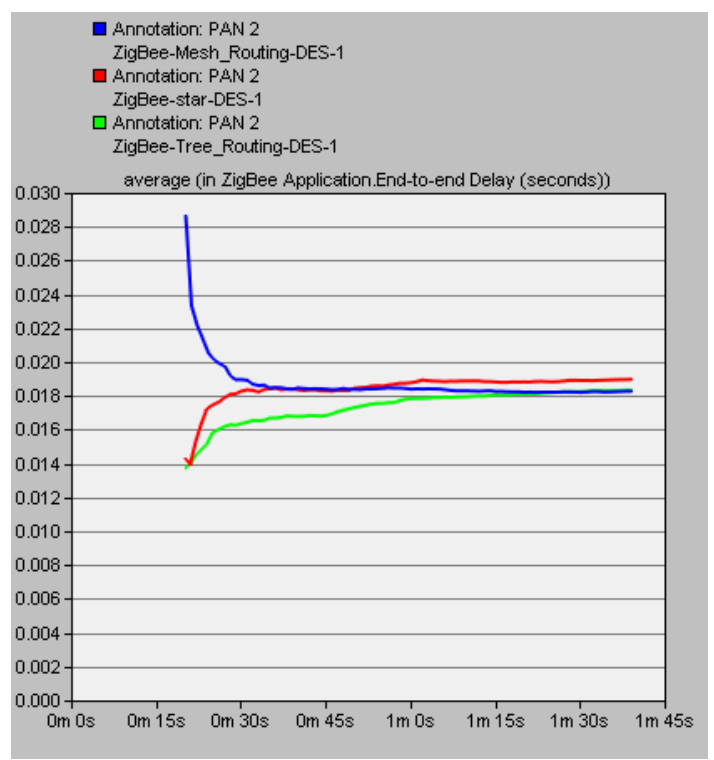

Fig. 6. ZigBee application layer end-to-end delays for various network topologies.

Results indicate that throughput is maximum in tree topology; this topology has fully functional devices and each cluster controls their own networks separately before communicate with others. This reduces collisions and retransmissions times. Mesh topology seems to be the best for having the highest throughput. This is suitable in situations where there are large numbers of nodes on the network.

Future work will include multiple clusters of traffic lights to coordinate traffic flow in large areas ultimately covering the whole metropolitan area. Information flow between multiple and sparsely distributed clusters will be realized through network routers.

\section{CONCLUSIONS}

A laboratory based PLC/SCADA, and a wireless network has been set up to study characteristics of traffic flow in arterial urban roads. Results from this study shows that the traffic lights can be synchronized by using PLC/SCADA systems, since they are capable of exchanging information about traffic load conditions from the nearby traffic light sets. Synchronization leads to continuous flow of vehicles in an elastic string mode. It has been found that Wireless Tree Network gives lower end-to-end delays. Mesh networks is best for data throughput in comparison to star networks. This is finding is important in critically safety related operations such as the traffic light controls. The system proposed here can be extended to intelligent transport systems and studying largescale traffic flow covering entire metropolitan areas. 


\section{REFERENCES}

[1] Traffic lights cause crushes, 2004, http://www.ncpa.org/sub/dpd/index.php?Article_ID=1119, last accessed on 22 February 2013.

[2] W. Cheewapattananuwong, P. Taneerananon, and T. Nakatsuji, "Mitigating traffic congestion and accidents in Thailand with intelligent transportation technology," Transportation Research Record, n. 2239, pp. 41-55, 2011.

[3] B. Sheffield, "Radio system controls railroad in Venezuela," Electronics, vol. 29, no. 12, pp. 158-16, 1956

[4] D.E. Boyce, "Combining communication and transport technology to improve urban travel choices," Information Technology: Social and Spatial Perspectives. Proceedings of an International Conference and its Impact on the Urban-Environmental System, 1988, pp. 141-52.

[5] H. Eren, Wireless Sensors and Instruments Networks, Design and Applications. Taylor and Francis Group: Boca Raton, 2006.

[6] L. Xiao, X. Peng, Z. Wang, B. Xu, and P. Hong, "Research on traffic monitoring network and its traffic flow forecast and congestion control model based on wireless sensor networks," 2009 Internationa Conference on Measuring Technology and Mechatronics Automation (ICMTMA), 2009, pp. 142-7.

[7] M.Y. Khalil, N.J. Al-Karaki, and M.A. Shatnawi, "Intelligent traffic light flow control system using wireless sensors networks" Journal of Information Science and Engineering, vol. 26, no. 3, pp. 753-768, 2010.

[8] Y. Huabing, and Mei Guo, "A self-adapting data collection approach in wireless sensor networks for urban traffic information monitoring," 2010 IEEE International Conference on Intelligent Computing and Intelligent Systems (ICIS 2010), pp. 196-200.

[9] H. Eren, and E. Fadzil, "Technical challenges for wireless instrumen networks - a case study with ZigBee," Proceedings of the 2007 IEEE Sensors Applications Symposium, pp. 1-6, 2007.

[10] R. Jordan, and C.A. Abdallah, "Wireless communications and networking: an overview," Report, Elect. and Comp. Eng. Dept., Univ. New Mexico, 2002.

[11] C.D.M. Papageorgiou, V. Dinopoulou, A. Kotsialos, and Y. Wang, "Review of road traffic control strategies," Proceedings of the IEEE, vol. 91, no. 12, pp. 2043-67, 2003.

[12] W.H. Xinkai, and G. Douglas, "Identification of oversaturated intersections using high-resolution traffic signal data." Transportation Research. Part C, Emerging Technologies, 2010, vol. 18, no. 4, pp. 626638.

[13] M.S.P. Taylor, "Exploring the nature of urban traffic congestion: concepts, parameters, theories and models," Proceedings - Conference of the Australian Road Research Board, vol. 16, no. 5, pp. 83-105, 1992.

[14] T. Sattel, and T. Brandt, "From robotics to automotive: Lane-keeping and collision avoidance based on elastic bands," Vehicle System Dynamics, vol. 46, no. 7, pp. 597-619, 2008.

[15] AlGhamdi, and H. Eren, "Microscopic analysis of highway congestion by DTCA model," International Journal of Modeling and Optimization, April 2012, vol. 2, no. 2, pp. 136-140.

[16] L. Yang, and C.X. Feng, "Design of traffic lights controlling system based on PLC and configuration technology," Proceedings of the International Conference on Multimedia Information Networking and Security, pp. 561-3, 2009.

[17] D.S. Zavalishchin, and G.A. Timofeeva, "Mathematical modeling of vehicle flow on a crossroads," IEEE International Conference on Control Applications, pp. 849 - 852, 2009.
[18] R.J. Duo Sun, B.H. Wang, "Timing of traffic lights and phase separation in two-dimensional traffic flow," Computer Physics Communications, vol. 181, no. 2, pp. 301-304, 2010.

[19] M. Brackstone, and M. McDonald, "Driver behavior and traffic modeling. Are we looking at the right issues?" IEEE IV2003 Intelligent Vehicles Symposium. Proceedings, pp. 517-21, 2003.

[20] A.C. Soh, M. Khalid, M.H. Marhaban, and R. Yusuf, , "Improving fuzzy traffic controller for multilane-multiple intersection," IEEE International Conference on Control and Automation, pp. 1819-1824, 2009.

[21] R.B. Elmar Brockfeld, A. Schadschneider, M. Schreckenberg, "Optimizing traffic lights in a cellular automaton model for city traffic," IEEE: 1 - 13, 2008.

[22] M. Patel, and N. Ranganathan, "IDUTC: An intelligent decision-making system for urban traffic-control applications," IEEE Transactions on Vehicular Technology, 2001, vol. 50, no. 3, pp. 816-29.

[23] L.G. Zhang, and Y.Z. Chen, "Hybrid petri net modeling of traffic flow and signal control," IEEE Proceedings of the 7th International Conference on Machine Learning and Cybernetics, ICMLC, 2008, vol. 4, pp. 2304-2308.

[24] L. Pengfei, and L. Jiakun, "Application of communication and remote control in PLC based on ZigBee technology," Proceedings of the 2009 International Conference on Computational Intelligence and Security (CIS 2009), pp. 533-6.

[25] G. Fang, and G. Zheng, "Research on PLC simulation control apparatus for intelligent traffic lights," Advanced Materials Research, 2012, vol. 482-484, pp. 805-811.

[26] M. Tubaishat, Q. Qi, Y. Shang, and H. Shi, "Wireless sensor based traffic light control," $5^{\text {th }}$ IEEE Consumer Communications and Networking Conference, CCNC 2008, pp. 702-706.

[27] J.F. Song, A.N. Bai, and R. Xue, "A reliable counting vehicles in traffic flow monitoring," Proceedings- $4^{\text {th }}$ International Conggress on Imag and Signal Processing, CISP 2011, pp. 522-524.

[28] Z. Liang, X. Qu, and X. Zhao, "Exploration based on ZigBee traffic signals area control system," Applied Mechanics and Materials, vol 135-136, pp. 535-539, 2012

[29] G.L. Foresti, and L. Snidaro, "Vehicle detection and tracking for traffic monitoring," Proceedings of Image Analysis and Processing ICIAP $13^{\text {th }}$ International Conference, 2005, pp. 1198-1205.

[30] M. Wójcikowski, R. Zaglewski, B. Pankiewicz, M. Kosowski, and S. Szczepanski, "Hardware-software implementation for sensor network for city traffic monitoring using FPGA and ASIC based sensor nodes," Journal of Signal Processing Systems, 2012, pp. 1-17.

[31] J. Alonso, J. Godoy, R. Sanz, E. Onieva, V. Milanes, J. Villagra, C. Gonzales, T. de Pedro, and R. Garcia, "Traffic light intelligent regulation using infrastructure located sensors," Computer Aided Systems Theory - EUROCAST, $13^{\text {th }}$ International Conference 2011, pp. 398-403.

[32] B. Zhou, J. Cao, X. Zeng, and H. Wu, "Adaptive traffic light control in wireless sensor network-based intelligent transport system," Proceedings of $72^{\text {nd }}$ Vehicular Technology Conference, 5 p, 2010.

[33] P. Jurcik, A. Koubaa, M. Alves, E. Tovar, and Z. Hanzalek, "A simulation model for the IEEE 802.15.4 protocol: Delay/throughput evaluation of the GTS mechanism," in Modeling, Analysis, and Simulation of Computer and Telecommunication Systems, 2007. MASCOTS '07, pp. 109-116. 